\title{
Compact and Highly Efficient Grating Couplers Between Optical Fiber and Nanophotonic Waveguides in Bonded InP-membranes
}

\author{
F. Van Laere (1), M. Ayre (2), D. Taillaert (1), D. Van Thourhout (1), T. F. Krauss (2) and R. Baets (1) \\ 1 : Ghent University - IMEC, Department of Information Technology \\ St. Pietersnieuwstraat 41,9000 Gent, BELGIUM \\ e-mail : frederik.vanlaere@intec.ugent.be \\ 2: School of Physics and Astronomy, University of St. Andrews, \\ St. Andrews, Fife, KY169SS \\ e-mal: ma37@st-andrews.ac.uk
}

\begin{abstract}
We present compact and highly efficient grating couplers in bonded InP-membranes for coupling between single-mode optical fiber and narophotonic waveguides. We have measured $56 \%$ coupling efficiency on grating couplers extended with a gold bottom mirror.
\end{abstract}

\section{Introduction}

Nanophotonic waveguides and components are the route towards large scale integration of photonic circuits. However, coupling light between single-mode fiber and nanophotonic waveguides remains an important problem. Key features of an effective solution to this coupling problem are low insertion loss, broadband operation, relaxed tolerances to fabrication and alignment. These requirements can be met by using an inverse taper [1]. This approach however does not allow for wafer-scale testing. We use grating couplers for coupling light out-of-plane from standard single-mode fiber to thin-film waveguides. In this approach, there is no need for cleaved facets and light can be coupled in and out everywhere on the chip, opening the prospect of wafer-scale testing. The grating is followed by an inplane taper to couple to single-mode nanophotonic waveguides. The principle is shown in Fig. 1

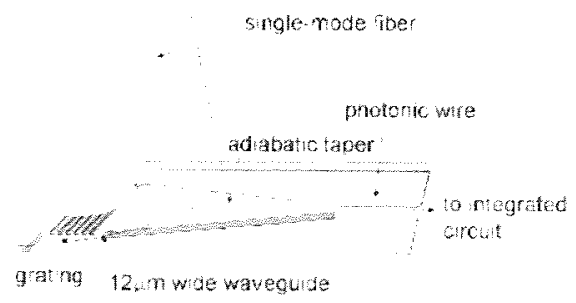

Fig. 1. Coupling principle for out-of-plane coupling between single-mode fiber and photonic wires.

When using a high refractive index contrast, these gratings can be very compact and relatively broadband. A coupling efficiency of $33 \%$ from fiber to waveguide was measured for $10 \times 10 \mathrm{~mm}^{2}$ grating couplers in SOI [2]. The $1 \mathrm{~dB}$ bandwidth is $40 \mathrm{~mm}$ and the alignment tolerances are relaxed $(2 \mathrm{um}$ for $1 \mathrm{~dB}$ excess loss). A 2D-grating version can be used for getting polarization independence through polarization diversity [3]. The coupling efficiency to fiber is limited by radiation towards the substrate however. In [4], we avold this by adding a bottom mirror to existing SOl-grating couplers and the measured coupling efficiency increases to $69 \%$. However, SOl is not very well suited for implementing active functionality. Therefore, we have transferred the principle to InP-based material. We apply wafer bonding for achieving high vertical index contrast membranes, which are important for ultra compact photonic circuits (e.g. high-Q microcavities). In [5], we have demonstrated $30 \%$ coupling efficiency between single-mode fiber and waveguides in BCB-bonded InP-membranes using grating couplers. In this paper, we report on the improvement of these InP. membrane grating couplers by adding a boltom mirror. The measured coupling efficiency increases to $56 \%$

\section{High vertical index contrast by wafer bonding}

We focus on adhesive bonding, using an intermediate low-index BenzoCycloButene (BCB)--layer (a spin-on polymer). The bonding procedure is shown in Fig. 2 . a.
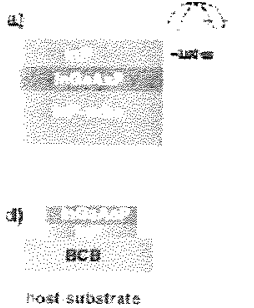

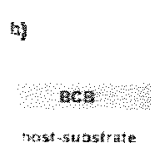

$e^{2}$

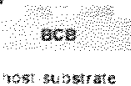

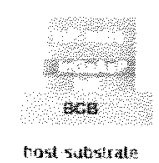

Fig.2. BCB-bonding procedure

We stant from an InP-based layer structure with an InP-substrate, an InGaAsP etch-stop layer and an InP-membrane layer. This structure is bonded by means of BCB onto a host-substrate. Afterwards, the original InP-substrate is removed, and finaly the etchstop layer is removed.

\section{Design}

The couplers are designed with CAMFR [6] for TEpolarization. The design method is described in delail in [7]. We use near vertical coupling at 10 degrees in order to avoid second order reflection. A gold bottom mirrot is added to the structure bo avoid radition 
towards the substrate. The period of the optimised grating is $660 \mathrm{~nm}$, the etch depth is $70 \mathrm{~nm}$ and the duiy cycle $50 \%$. The thickness of the BCB bonding layer is optimised in order to achieve constructive interference between the direcly upwards radiated wave and the reflected wave at the bottom mirror. The reflected wave also interacts with the grating and the BCB-layer thickness influences the coupling length of the grating. The maximum coupling efficiency as a function of BCB-thickness is shown in Fig. 3. The optimal thickness is $1.23 \mu \mathrm{m}$ and the maximum coupling efficiency is $78 \%$. A field plot of the optimal structure is shown in Fig 4

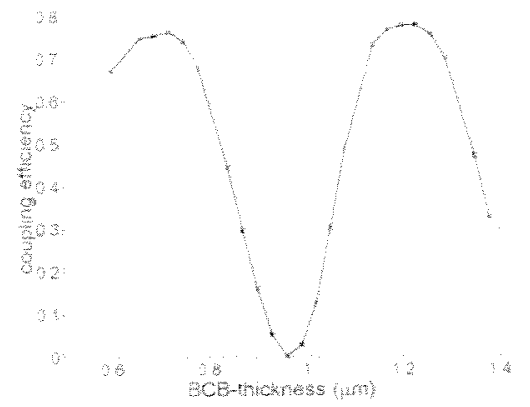

Fig. 3. Maximum coupling efficiency as a function of $B C B$-thickness.

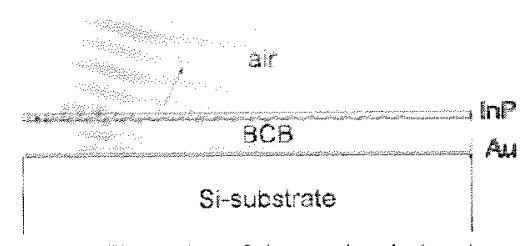

Fig. 4. Field plot of the optimal structure.

\section{Fabrication}

The layer structure consists of an InP-substrate, a Q1.22 etch-stop layer and a $300 \mathrm{~nm}$ inP-membrane layer. First, the gratings and waveguides are defined by e-beam lithography using PMMA, and transferred to a $\mathrm{SiO}_{2}$ hard-mask by Reactive lon Etching (RIE). The structures are etched to a targeted depth of 70 nm into the InP-heterostructure by Inductive Coupled Plasma (ICP) etching. This structure is then bonded using $B C B$ onto a gold coated Si-substrate, with the grating at the bottom side. The BCB-layer thickness is targeted to $1.23 \mu \mathrm{m}$. The $\mathrm{BCB}$ is cured for 1 hour at 250 degrees in a nitrogen environment. Afterwards, the substrate is removed using lapping and wet etching. Finally the etch-stop layer is removed by wet etching.

\section{Measurements}

The coupling efficiency is determined from a fiber-tofiber transmission measurement for TE-polarization. The structure consists of an input coupler, a $12 \mathrm{~mm}$ wide waveguide and an output coupler. We assume that input and output coupler are the same. By characterizing losses in our setup, we calculate the coupling efficiency to fiber from the transmission efficiency. The measurement result is shown in Fig. 5 , together with the simulated curve. The measured coupling efficiency is $56 \%$ and the $1 \mathrm{~dB}$ bandwidth is around $45 \mathrm{~nm}$. The deviation between theory and experiment is caused by a deviation in theoretical and fabricated structure (etch depth, filling factor, BCBthickness).

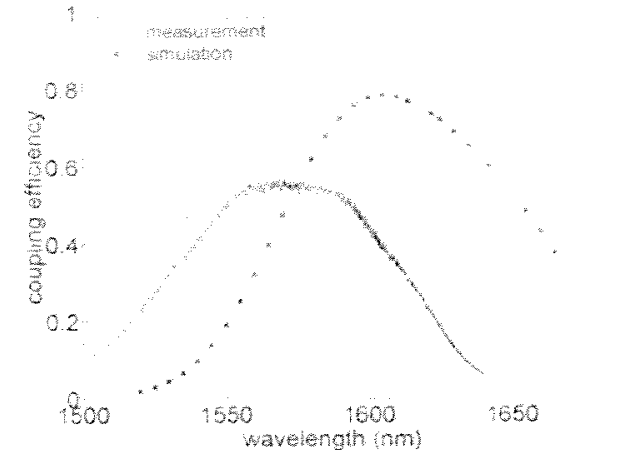

Fig. 5. Measured and simulated coupling efficiency.

\section{Conclusions}

We have designed and fabricated compact and highly efficient grating couplers between single mode fiber and waveguides in $\mathrm{BCB}$-bonded InP-membranes. A gold bottom mirror is used to increase the coupling efficiency to a measured value of $56 \%$. Apodising the grating can increase the coupling efficiency further to a theoretical value over $90 \%$ [6].

\section{Acknowledgements}

This work was supported in part by the European Union through the IST-FUNFOX project and the Network of Excellence ePIXnet and by the Belgian IAP-PHOTON Network. F. Van Laere thanks the Institute for the Promotion of innovation through Science and Technology in Flanders (IWT) for a specialisation grant. Alcatel-Thales $I I-V$ Lab is acknowledged for providing the inP epi-wafers.

\section{References}

1 T. Tzuchizawa et al, J. Select. Top. Quant. Elect. Vol. 11 (2005), pp. 232-240

2 D. Tallaert et al, Proc of 2005 IEEELLOS Workshop on Fibres and Optical Passive Components, pp 115-120

3 D. Tallaert et al, PTL, Vol 15 (2003), pp.1249-1251

4 F. Van Laere et al. OFC (2006). PDP15

$5 \mathrm{~F}$. Van Laere et al, Electronics Letters, Vol. 42 (2006), pp. $343-345$

6 Bienstman et al, Optical and Quantum Electronics. Vol. $33(2001)$, pp. 327-341

7 D. Tallaert ef al, Optics Letters, Vol. 29 (2004), pp $2749-2751$ 


\section{$24-28$ September 2006 ,} Cannes, France

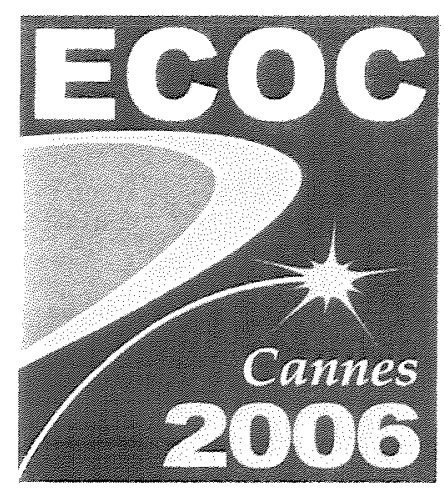

The SEE (Societé de IElectricité, de l'Electronique et des Technologies de finformation et de la Communication)

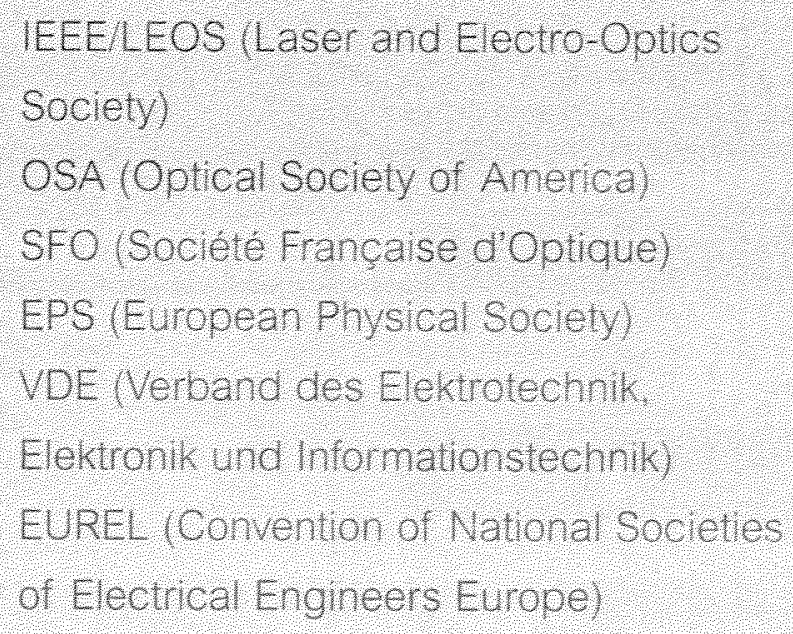




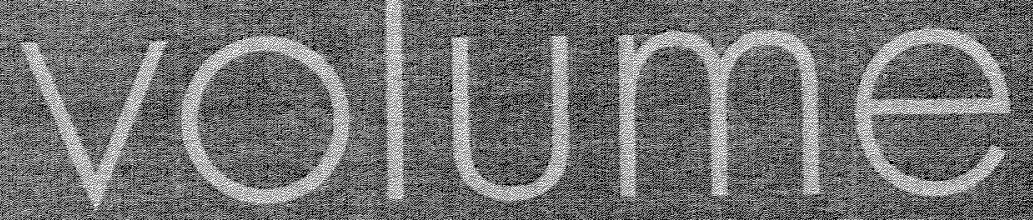

PROG EFEIIINCS

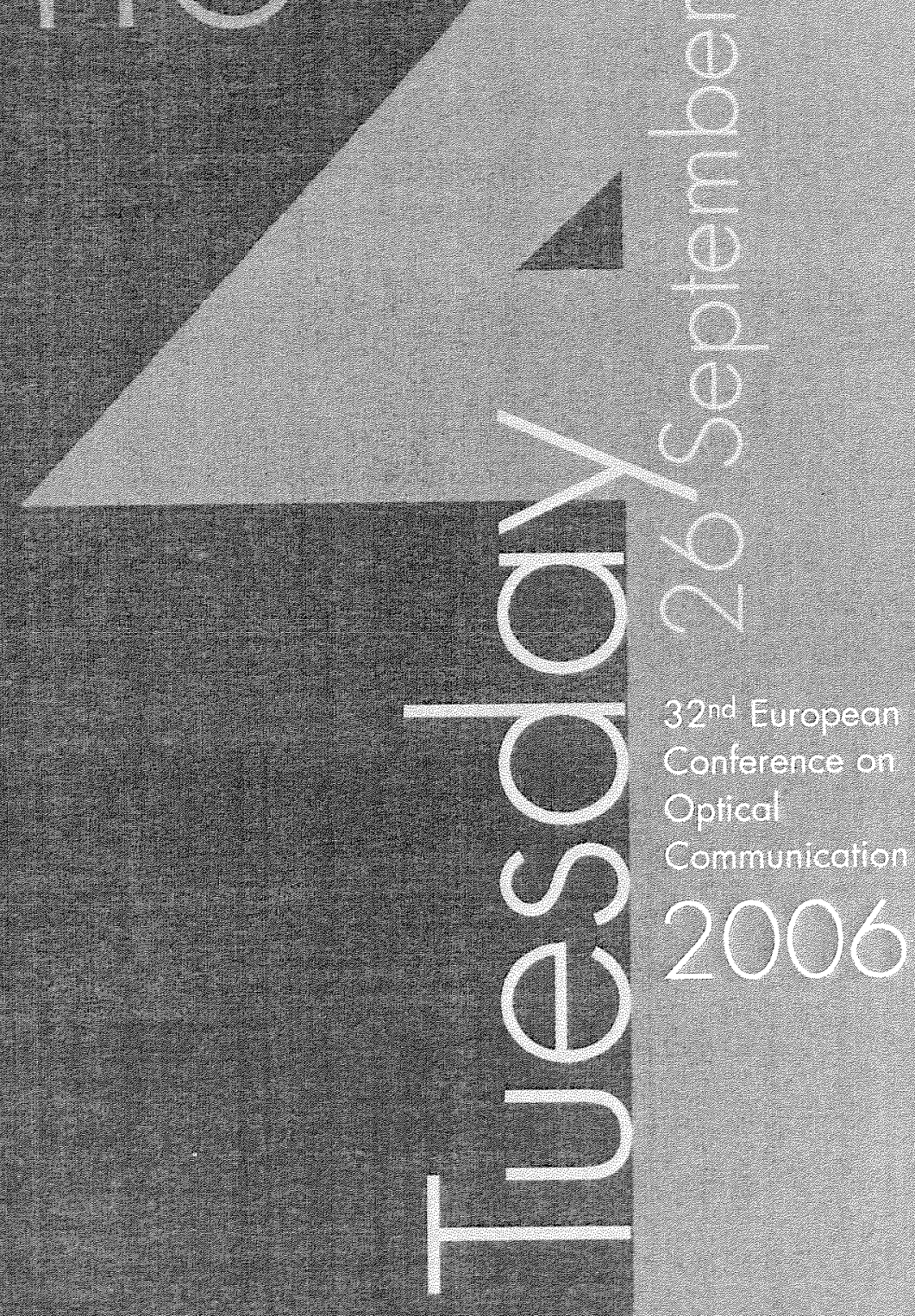

[न्रण

COMT:

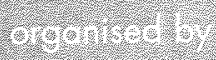

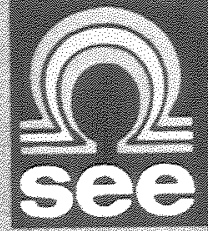

1413 


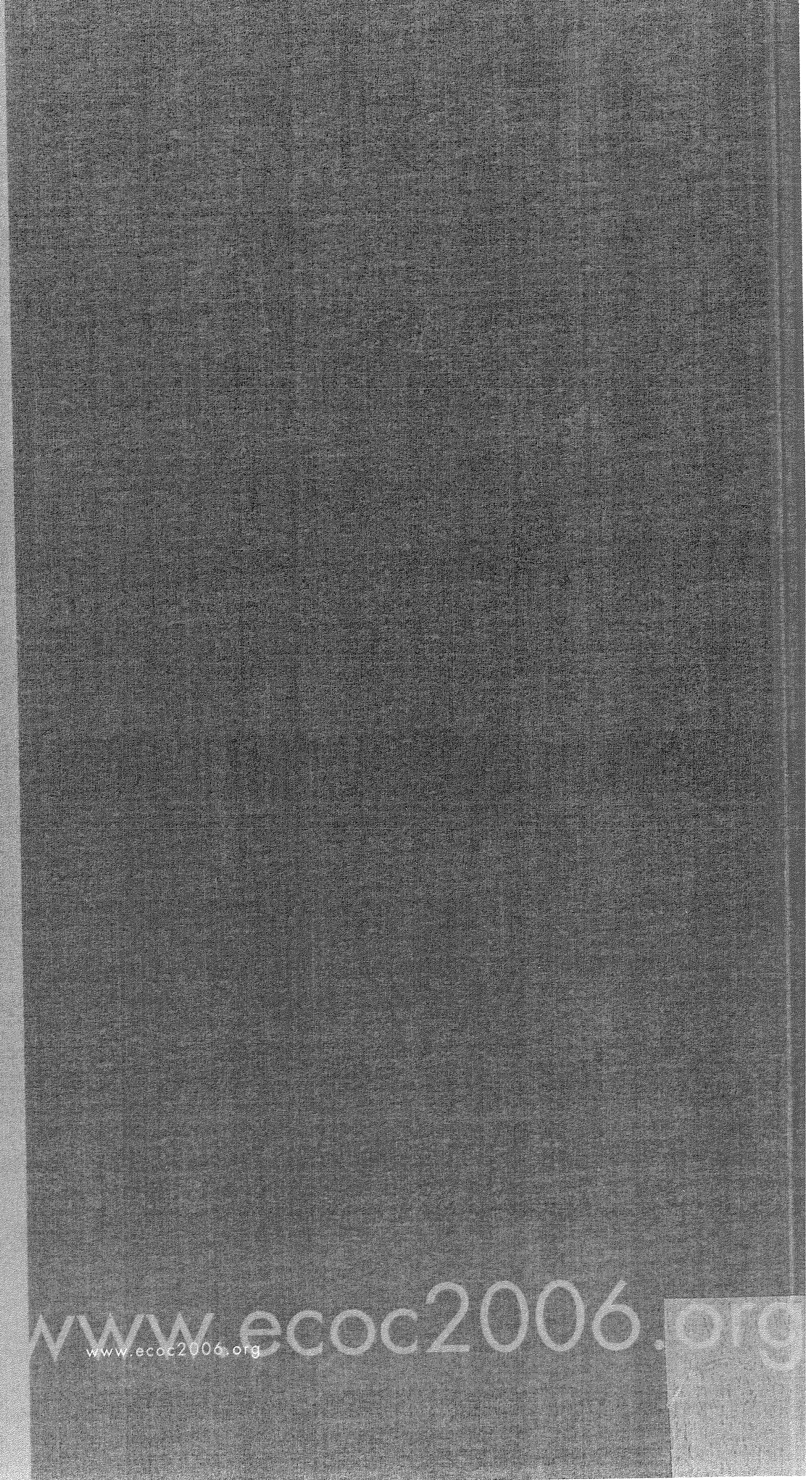

